\title{
Leg Length Discrepancy Due to Loss of Femoral Antecurvatum After Elastic Stable Intramedullary Nailing of Diaphyseal Fractures of the Femur in Children
}

\author{
Panagiotis V. Samelis ${ }^{1,2}$, Eftychios Papagrigorakis ${ }^{3}$, Theodore Troupis ${ }^{4}$, Panagiotis Koulouvaris ${ }^{5}$ \\ 1. Orthopaedics, Children's General Hospital Panagiotis \& Aglaia Kyriakou, Athens, GRC 2. Orthopaedics, Orthopaedic \\ Research and Education Center Attikon Hospital, Athens, GRC 3. Orthopaedics, KAT Trauma Hospital, Athens, GRC 4.
} Surgery, University of Athens Medical School, Athens, GRC 5. Orthopaedics, Attikon University Hospital, Athens, GRC

Corresponding author: Panagiotis V. Samelis, samelis_takis@yahoo.com

\begin{abstract}
Limb length discrepancy (LLD) is a frequent complication after elastic stable intramedullary nailing (ESIN) of femoral shaft fractures in children. It is the result of either shortening or lengthening of the affected limb. A shorter limb is usually observed when there is no strict adherence to the main indication of the technique, which is a transverse or short oblique fracture of the diaphysis. A longer limb may be the result of either improper reduction and significant dissociation of fracture fragments, or stimulation of growth of the fractured bone of the child, known as overgrowth. We describe a potential third cause of limb lengthening after treating femoral shaft fractures with the ESIN technique. LLD may be the result of acute femur lengthening due to the loss of normal femoral antecurvatum.
\end{abstract}

Categories: Orthopedics

Keywords: children, elastic, intramedullary, femur, fracture, discrepancy, limb, length, antecurvatum, nail

\section{Introduction}

Elastic stable intramedullary nailing (ESIN) using a pair of titanium nails is a widely accepted method of treatment of long bone diaphyseal fractures in children, leading to fast recovery, short hospital stay, and fewer complications compared with other methods of treatment [1-4]. Opposite to typical rigid intramedullary nailing for fractures of the femur, ESIN enters the femoral diaphysis not through, but between the growth plates of the long bone, thus avoiding potential growth disturbance secondary to physis injury and avascular necrosis of the femoral head secondary of injury of the nutrient vessels of the femoral head $[1,5]$. Unfortunately, this is accomplished in expense of fracture stability and less control on the mechanical and torsional profile of the affected limb. Fortunately, the rapid healing and remodelling potential of the growing skeleton of the child compensates minor deviations from normal [1].

Received 11/30/2019

Review began 12/02/2019 Review ended 12/08/2019 Published 12/11/2019

\section{○ Copyright 2019}

Samelis et al. This is an open access article distributed under the terms of the Creative Commons Attribution License CC-BY 3.0., which permits unrestricted use, distribution, and reproduction in any medium, provided the original author and source are credited.
The main indication of ESIN is a transverse or short oblique long bone diaphyseal fracture of the growing skeleton in children between the ages of five and 12 years and weighing less than $50 \mathrm{~kg}$ [6]. Due to the simple operative technique and the rapid healing potential of the growing skeleton of the child, surgeons increasingly use this technique even in oblique or comminuted fractures $[1,5,7]$.

The ESIN technique is quite simple; under image intensification, the nails are inserted from opposite sides of the distal femoral metaphysis, advanced towards the fracture, and anchored to the proximal femoral metaphysis after fracture reduction. Symmetric bending of the elastic nails prior to insertion is an integral part of this technique, in order, in terms of biomechanics, to obtain three-point fixation between each nail and the bone [8].

Complications such as skin problems due to prominent hardware, infection, fracture nonunion, hardware failure (bending, breakage) and complications during hardware removal may be observed in children treated with the ESIN technique $[2,8]$. The complications rate is higher in more complex fractures and in children weighing more than $40 \mathrm{~kg}[5,9]$.

Some complications are inherent with the ESIN technique. Until callus consolidation is complete, ESIN allows some movement (telescoping, bending, translation, torsion) at the fracture site. This may result in loss of longitudinal, torsional and angular alignment of the fracture and to subsequent malalignment and limb length discrepancy (LLD) [4-5].

The femoral shaft presents a normal anterior sagittal bow, termed antecurvatum. Femoral antecurvatum is the result of the intrinsic growing pattern and the extrinsic mechanical loading of the growing femur. It is present in the fetus and shows geographical and racial variance [10]. The normal radius of curvature of the 


\section{Cureus}

femur is approximately $120 \mathrm{~cm}+/-36 \mathrm{~cm}$ irrelevant of age [11]. Knowledge of the normal femoral antecurvatum is important in the typical rigid intramedullary nailing of femoral shaft fractures in adults, since the radius of curvature of the nail should correspond to the radius of anterior bowing of the femur, in order to avoid postoperative complications such as anterior femoral cortex penetration or hip and knee pain $[10,11]$.

In children, anatomic reduction of femoral shaft fractures should include the restoration of femoral antecurvatum as well. Current literature lacks information about the effect of ESIN on femoral antecurvatum. In clinical practice, changes of femoral antecurvatum are rather ignored, unless they are extreme and cause significant discomfort to the patient. However, it seems that ESIN fails to restore femoral antecurvatum. Immediate postoperatively and as long as the patient is bedridden, the femur may assume a straight configuration on the sagittal plane (loss of antecurvatum). Loss of femoral antecurvatum results in a straight femoral shaft in the sagittal plane and may lead to acute postoperative leg lengthening after treatment of stable (transverse, short oblique) diaphyseal fractures with the ESIN technique. Prolonged bed stay may turn this finding to a permanent malunion and, along with potentially missed fracture diastasis and overgrowth, contribute to LLD with a longer affected limb.

The aim of this study is to describe the loss of femoral antecurvatum as a potential complication of the treatment of femoral shaft fractures with the ESIN technique that may lead to LLD due to acute lengthening of the operated femur.

\section{Case Presentation}

An 11-year-old boy presented with a transverse fracture of his left femur after a fall from height (Figure 1). Surgical treatment using titanium nails according to the principles of the ESIN technique was decided. Traction table and image intensification were used. Nails of $3.5 \mathrm{~mm}$ width were chosen, corresponding approximately to $40 \%$ of the width of the narrowest diameter of the femoral shaft. Prior to insertion, the nails were bent three times the width of the medullary canal. The nails entered the medullary canal from opposite sides of the distal femoral metaphysis and were advanced retrogradely up to the fracture level. After closed fracture reduction, the nails were further advanced into the proximal fragment and anchored firmly into the proximal femoral metaphysis (Figure 2). Care was taken to obtain accurate reduction without a gap between the fracture fragments.

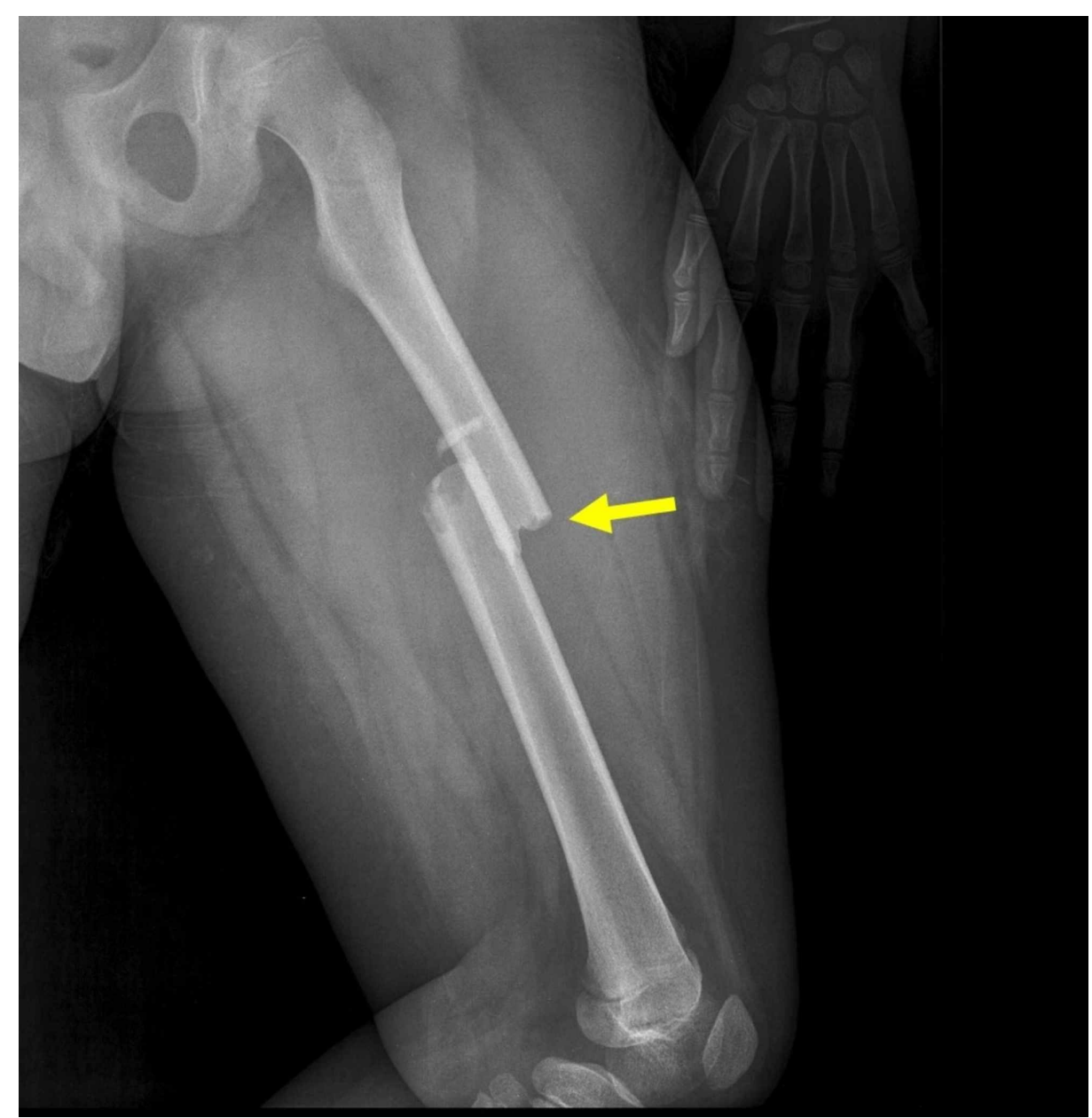




\section{Cureus}

FIGURE 1: Midshaft transverse/short oblique fracture with minimal comminution of the diaphysis of the left femur of an 11-year-old boy

Arrow indicates the fracture of the femur.

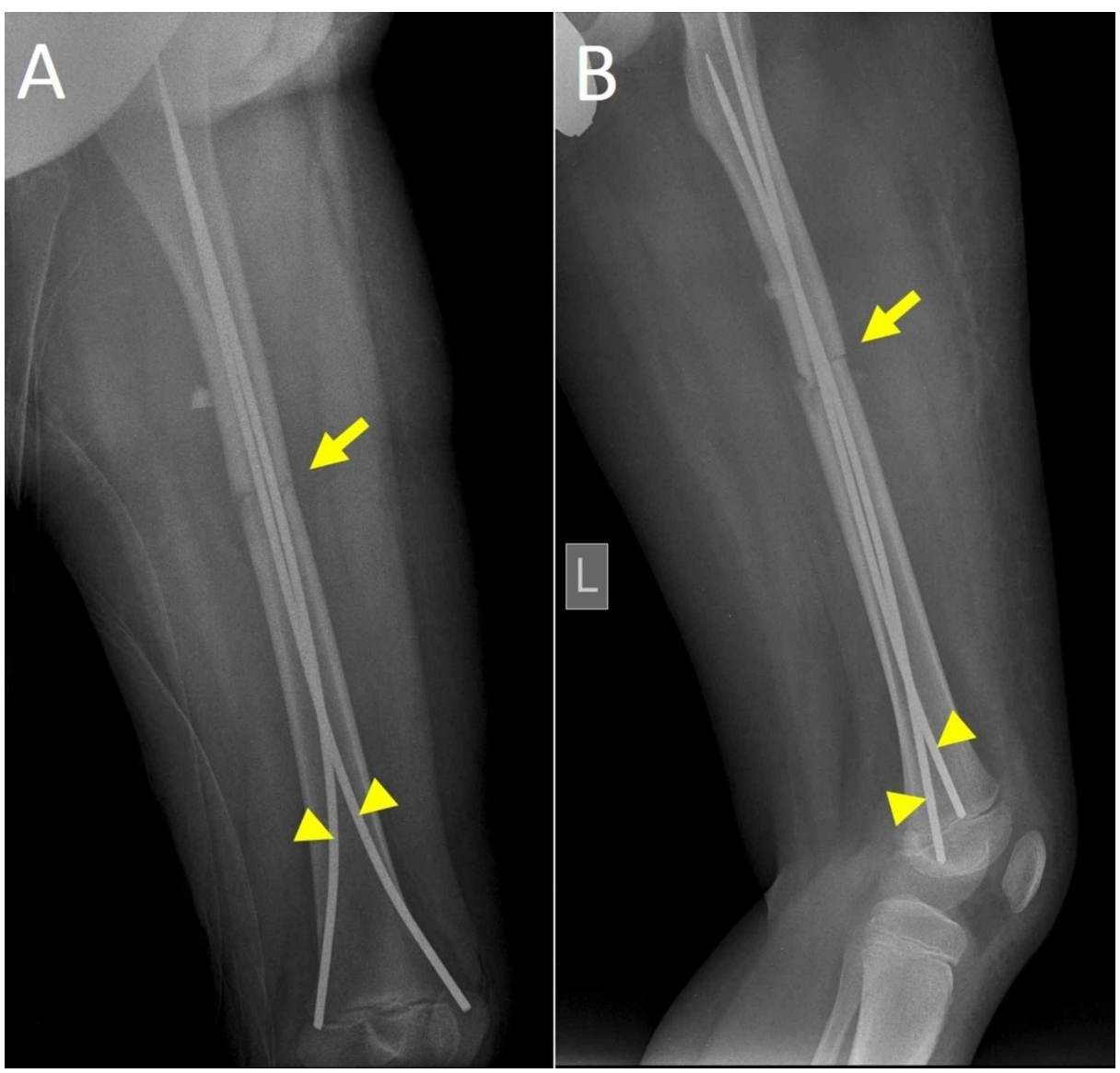

FIGURE 2: Postoperative X-ray after elastic stable intramedullary nailing (ESIN) of the left femur

A. Anteroposterior view, B. Lateral view.

Arrows indicate the fracture after reduction and osteosynthesis. Arrowheads indicate the titanium nails. Slight diastasis of the fracture fragments on both views is seen. Loss of femoral antecurvatum is evident on the lateral X-ray view.

After resuscitation and transfer of the patient from the traction table to the stretcher, LLD due to a longer fractured limb was evident. The operated limb was about $1.5 \mathrm{~cm}$ longer than the contralateral (clinical measurement). LLD was evident as long as the patient was supine (Figure 3). 


\section{Cureus}

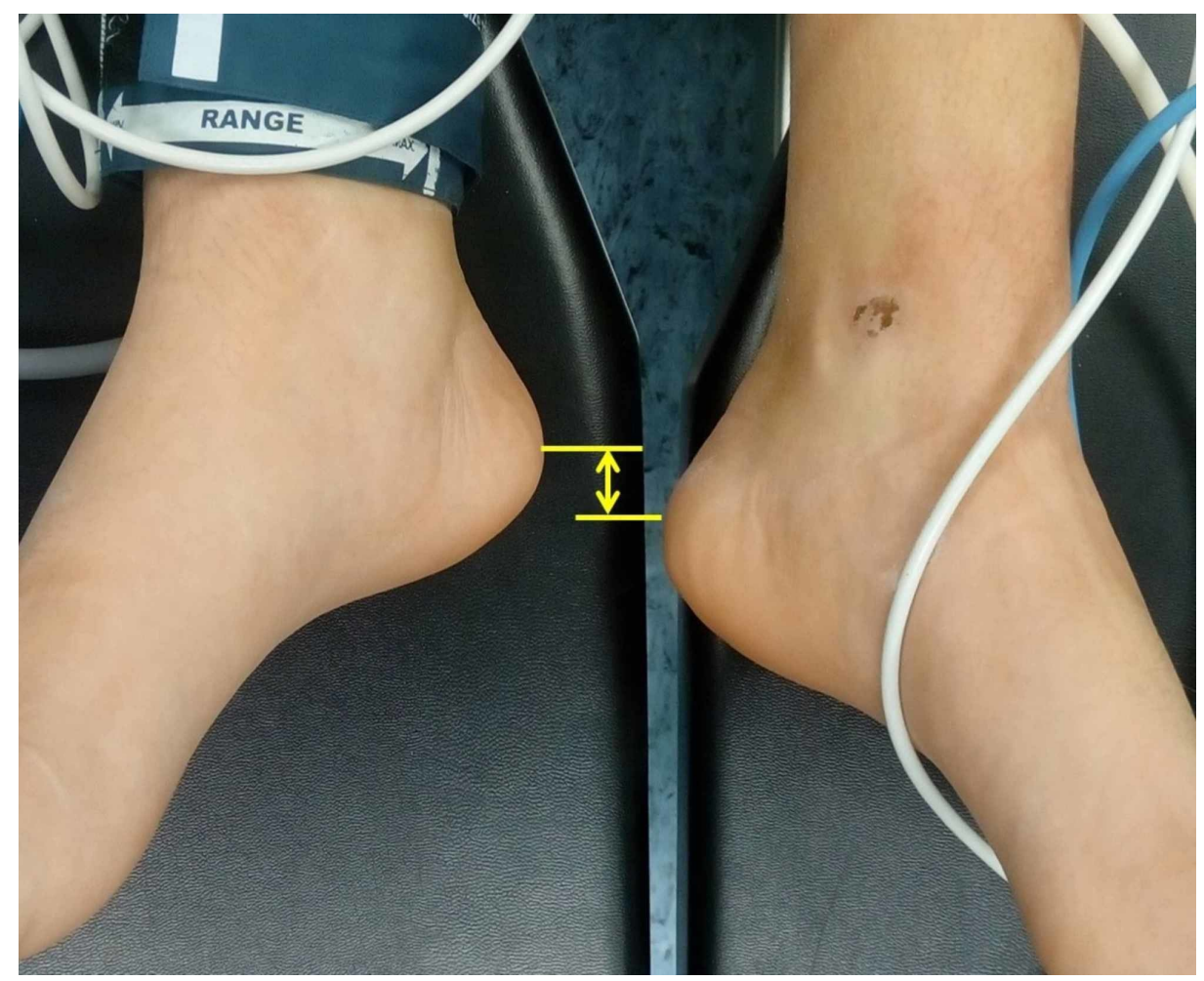

FIGURE 3: Immediate postoperative limb length discrepancy (LLD)

The operated left leg is longer than the contralateral. Arrow indicates LLD of approximately $1.5 \mathrm{~cm}$.

Follow up examinations focused on fracture healing and on LLD. Partial weight bearing as tolerated by pain was recommended. The patient adapted well to the initially longer limb.

The patient was dismissed one week postoperatively with instruction of partial weight bearing as tolerated for one month. Progressive weight bearing was recommended on follow-up examinations, according to radiologically confirmed callus formation. At three months postoperatively, fracture healing was complete and the patient was encouraged to resume noncompetitive sports. On clinical examination, slight ipsilateral knee recurvatum might be the only hint for the straightened femur. Three months after surgery, loss of femoral antecurvatum of the operated limb was still evident on the long limb standing lateral X-ray. LLD due to longer operated femur of $+1.24 \mathrm{~cm}$ (radiological measurement as seen in Figure 4) persisted, compared to the contralateral femur. The ipsilateral tibia, was $2 \mathrm{~mm}$ longer than the contralateral (radiological measurement); however, this finding was considered as non-significant for the total LLD. 


\section{Cureus}

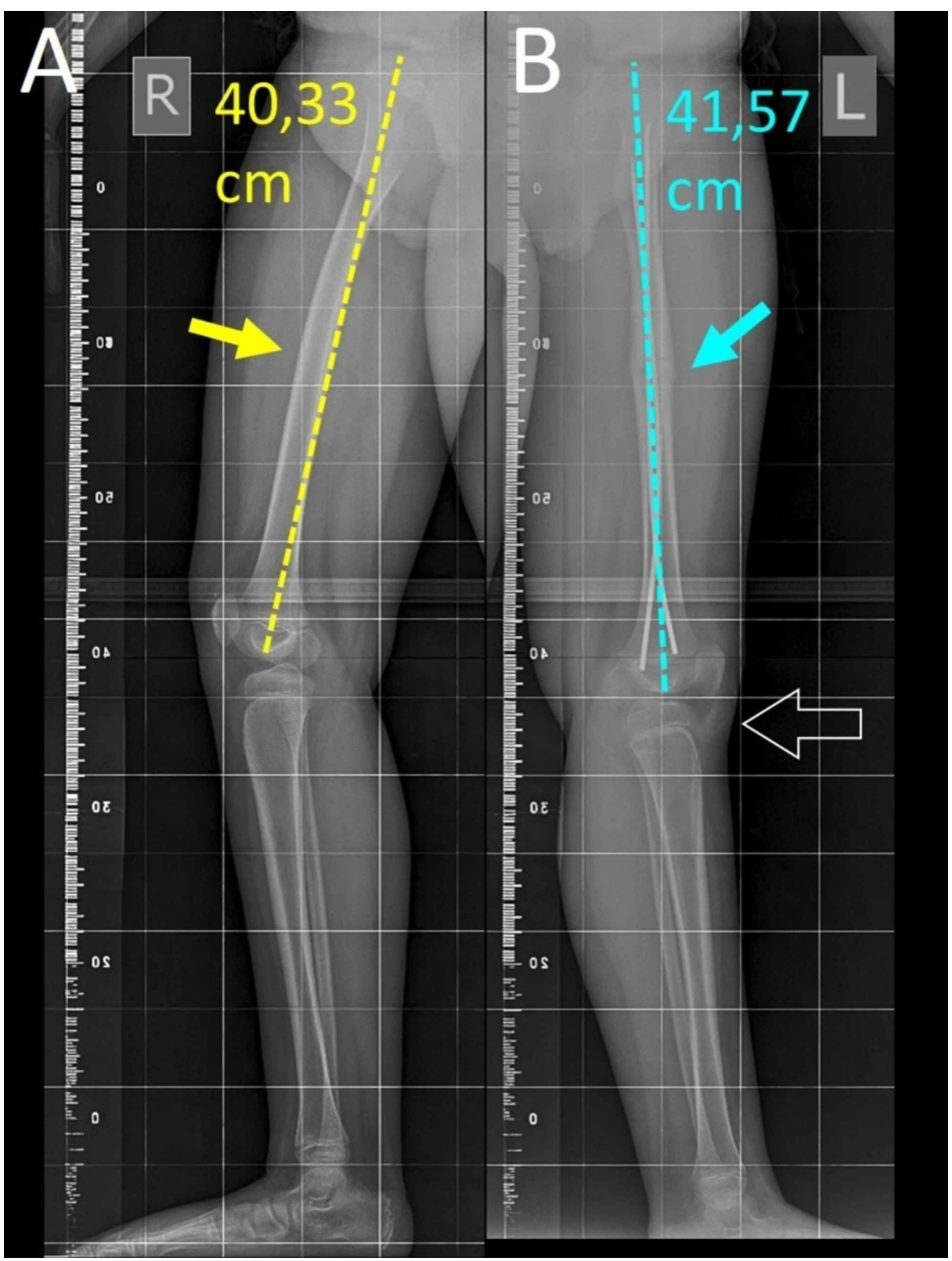

FIGURE 4: Long standing lateral X-ray view of the right $(A)$ and the left (B) femur three months after surgery

Limb length discrepancy with a longer left femur is obvious $(+1.24 \mathrm{~cm})$. A. Normal femoral antecurvatum of right femur (yellow arrow), B. The left femur is straightened due to loss of femoral antecurvatum (blue arrow). The left leg presents apparent (false) knee recurvatum secondary to loss of femoral antecurvatum (arrow outline).

Healing was complete at six months postoperatively. The patient was asymptomatic. Walking and participation in sports was unrestricted. Hardware removal was scheduled. A slight LLD of less than $5 \mathrm{~mm}$ was detected on clinical examination. Femoral antecurvatum was partially restored (Figure 5). 


\section{Cureus}

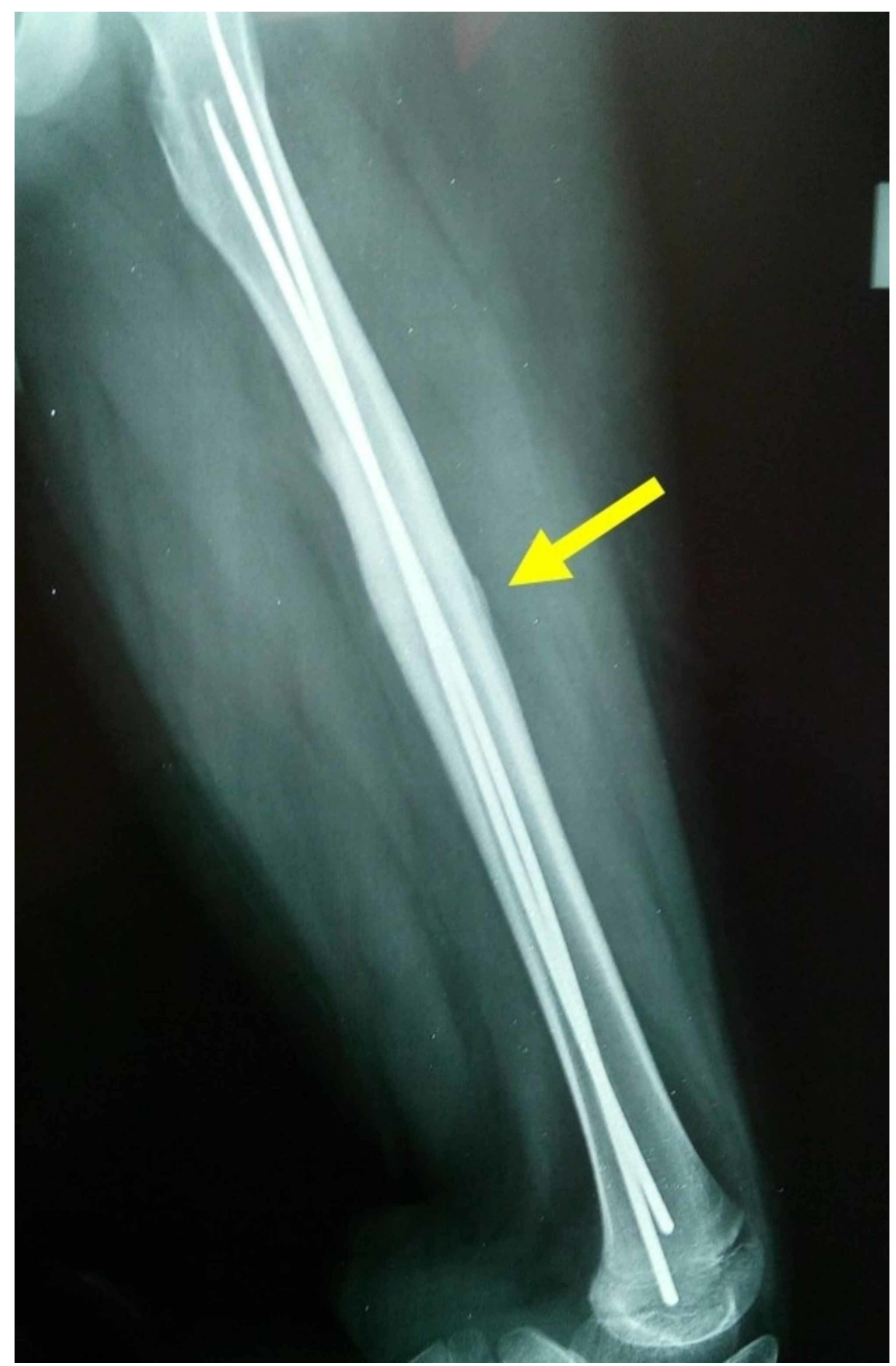

FIGURE 5: Lateral X-ray view of the operated left femur six months after surgery; fracture healing is complete

Arrow indicates complete fracture healing and a partially restored antecurvatum of the left femur.

\section{Discussion}

There are two types of LLD after ESIN of femoral shaft fractures in children:

The first type is limb shortening, seen with unstable fracture patterns (comminuted, spiral). Shortening of the fractured limb may be the immediate result of surgery (missed during surgery) or may be due to postoperative loss of reduction (telescoping of fracture fragments due to unstable osteosynthesis) [5]. Limb shortening after a diaphyseal long bone fracture is never attributed to growth deceleration of the injured bone and contrary to overgrowth, is always considered a complication. 


\section{Cureus}

The second type of LLD is a longer affected limb, that may be observed either immediately after surgery (early, acute lengthening) or may be a late result, known as overgrowth.

First described by Truesdell in 1921, overgrowth was attributed to stimulation of growth secondary to trauma. Most authors report an average overgrowth of 8-10 mm after femoral shaft fracture [12-14]. Overgrowth is not confined to the fractured femur, but, to a lesser extent, involves the non-fractured ipsilateral tibia as well [10-11]. It seems that acceleration of growth reaches its peak at three months postinjury and returns to normal after 40 to 60 months for tibial and femoral fractures respectively [11]. Other authors confine overgrowth within the healing period and support that the difference at the end of healing is permanent. It has been postulated that it is not necessary to strive for perfection of reduction of long bone fractures in children, because overgrowth and remodeling compensate well for minor deviations from normal [11]. Epiphysiodesis, in order to address overgrowth greater than $2 \mathrm{~cm}$, is rarely necessary [1,3].

Acute lengthening after a femoral shaft fracture treated with ESIN has two aspects. One aspect is fracture diastasis, that is, a gap is present between the main fracture fragments, that was missed and left uncorrected during surgery. However, acute lengthening may be the result of a previously not described complication of femoral shaft fractures in children, treated especially with the ESIN technique. This complication is not a late effect, but is rather an adverse effect of the ESIN technique on the normal femoral antecurvatum. The concept of how a change of the femoral antecurvatum after a midshaft fracture affects femoral length is shown in Figure 6.

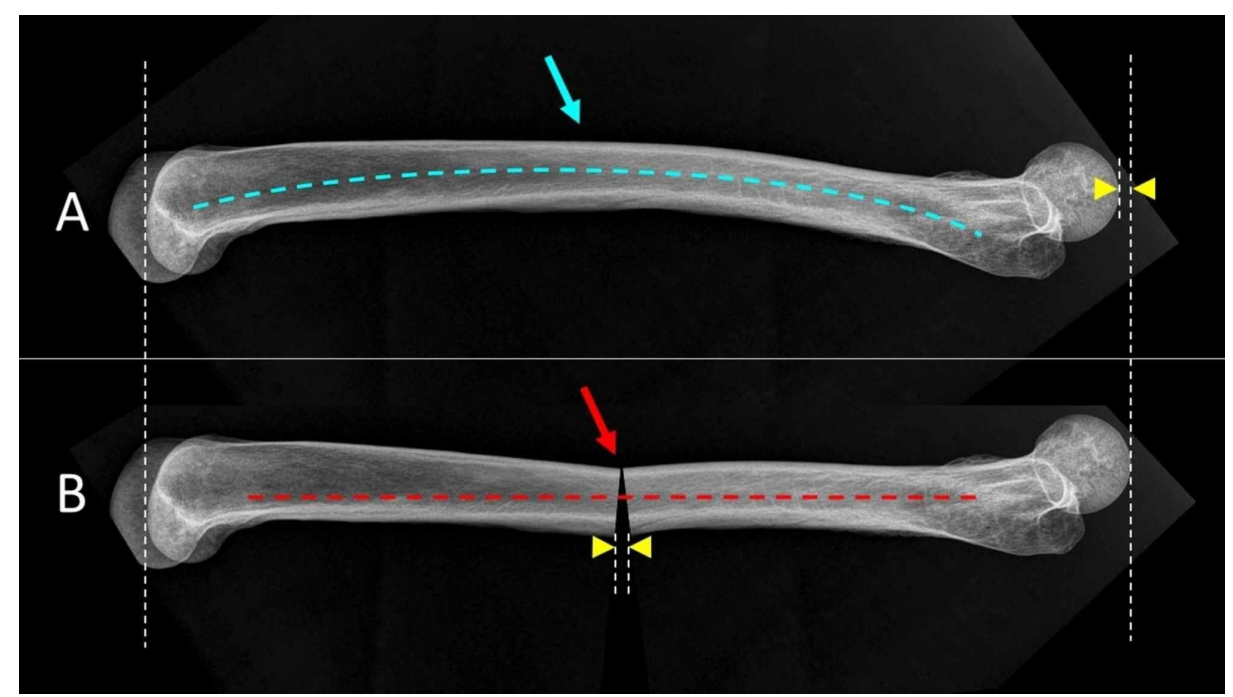

FIGURE 6: The effect of femoral antecurvatum on the length of the femur is shown on the lateral X-ray of a femoral bone

A. Normal femoral antecurvatum. B. Loss of femoral antecurvatum after ESIN

A. Blue arrow indicates normal femoral antecurvatum (blue dashed line), B. Red arrow indicates virtual transverse midshaft femoral fracture. Osteosynthesis with ESIN (red dashed line) leads to loss of the anterior curvature of the femur, either because the titanium nails are straight on the sagittal plane or secondary to the inherent sagittal instability of the bone-nail construct when treated with the ESIN technique. As a result, the femur becomes relatively longer than prior to the fracture (arrowheads).

ESIN: elastic stable intramedullary nailing.

In fact, the ESIN technique inherently fails to restore femoral antecurvatum after a femoral shaft fracture. This is because preoperative bending of the nails applies only in the frontal plane, thus providing some resistance only against varus-valgus angulation. On the contrary, the nails are straight in the sagittal plane and hence, either force the diaphysis to assume a straight configuration too (the femur is "stretched" by the nail) or fail to provide fracture stability against anteroposterior angulation. Thus, the femur-nail construct sags in the sagittal plane under the weight of the affected leg as long as the patient is bedridden.

Most studies consider deformities in the sagittal plane after ESIN of femoral shaft fractures (procurvatum, recurvatum) as late complications or malunions [5,15-16]. Few studies report on the immediate postoperative effect of ESIN on femoral antecurvatum. One study recommends the insertion of a third titanium nail in order to reinforce the bone-implant construct against deformation in the sagittal and frontal plane [5]. Other studies support that femoral antecurvatum is either spontaneously restored at three weeks after surgery or is not significantly affected by ESIN $[1,8]$. However, immediate postoperative (early) 
loss of femoral antecurvatum after ESIN of femoral shaft fractures is obvious on radiographs presented in several studies, but this finding is not commented on [17-19]. One of these studies shows the X-rays of a patient where it is evident that the immediate postoperative loss of femoral antecurvatum remains unchanged at one and 10 months postoperatively [19].

Interestingly, some studies report that transverse femoral fractures in children, treated using ESIN, have the highest tendency for posttraumatic lengthening compared with comminuted or spiral fractures $[1,4]$. This is consistent with the presented case and at least some part of the LLD could be attributed not only to overgrowth or to a postoperative fracture gap, but also to potentially decreased femoral antecurvatum after ESIN of transverse femoral shaft fractures.

Gulati et al. did not find significant impact of sagittal angulation of the femur on the final limb length after conservative treatment of femoral shaft fractures in children younger than 10 years of age [20]. To our knowledge, most studies that deal with LLD after femur fractures, treated with the ESIN technique, do not take into account the potential impact of sagittal angulation of the femur on final limb length. Thus, the validity of published data about overgrowth and LLD after a femoral shaft fracture treated with the ESIN technique is questioned, since the length of the femur after a fracture depends not only on overgrowth, but also on the adverse effect of ESIN on normal femoral antecurvatum.

\section{Conclusions}

Immediate postoperative limb lengthening secondary to loss of femoral antecurvatum is a benign and selflimiting complication after ESIN of femoral shaft fractures in children. This complication is inherent with the ESIN technique due to either the straight configuration of the titanium nails in the sagittal plane or posterior sag of the bone-nail construct. In the presented case, loss of femoral antecurvatum was almost corrected at hardware removal and the patient had an excellent outcome. Immediate postoperative loss of femoral antecurvatum may go undiagnosed in bilateral femoral fractures, as in polytrauma patients. Early ambulation and controlled weight bearing according to callus formation is strongly indicated, especially in stable fracture types of the femoral diaphysis (transverse, short oblique fractures), in order to progressively restore femoral antecurvatum and to avoid permanent lengthening of the affected femur, resulting in potentially significant limb length discrepancy.

\section{Additional Information}

\section{Disclosures}

Human subjects: Consent was obtained by all participants in this study. Conflicts of interest: In compliance with the ICMJE uniform disclosure form, all authors declare the following: Payment/services info: All authors have declared that no financial support was received from any organization for the submitted work. Financial relationships: All authors have declared that they have no financial relationships at present or within the previous three years with any organizations that might have an interest in the submitted work. Other relationships: All authors have declared that there are no other relationships or activities that could appear to have influenced the submitted work.

\section{References}

1. Ligier JN, Metaizeau JP, Prévot J, Lascombes P: Elastic stable intramedullary nailing of femoral shaft fractures in children. J Bone Joint Surg Br. 1988, 70:74-7. 10.1302/0301-620X.70B1.3339064

2. Flynn JM, Luedtke LM, Ganley TJ, et al.: Comparison of titanium elastic nails with traction and a spica cast to treat femoral fractures in children. J Bone Joint Surg Am. 2004, 86:770-7. 10.2106/00004623-20040400000015

3. Vierhout BP, Sleeboom C, Aronson DC, Van Walsum AD, Zijp G, Heij HA: Long-term outcome of elastic stable intramedullary fixation (ESIF) of femoral fractures in children. Eur J Pediatr Surg. 2006, 16:432-7. 10.1055/s-2006-924737

4. Salem KH, Keppler P: Limb geometry after elastic stable nailing for pediatric femoral fractures . J Bone Joint Surg Am. 2010, 92:1409-17. 10.2106/JBJS.I.00538

5. Kaiser MM, Stratmann C, Zachert G, Schulze-Hessing M, Gros N, Eggert R, Rapp M: Modification of elastic stable intramedullary nailing with a 3rd nail in a femoral spiral fracture model - results of biomechanical testing and a prospective clinical study. BMC Musculoskelet Disord. 2014, 8:3. 10.1186/1471-2474-15-3

6. Khoriati AA, Jones C, Gelfer Y, Trompeter A: The management of paediatric diaphyseal femoral fractures: a modern approach. Strategies Trauma Limb Reconstr. 2016, 11:87-97. 10.1007/s11751-016-0258-2

7. Soni JF, Schelle G, Valenza W, Pavelec AC, Souza CD: Unstable femoral fractures treated with titanium elastic intramedullary nails in children. Rev Bras Ortop. 2015, 4:575-80. 10.1016/S2255-4971(15)30006-9

8. Mazda K, Khairouni A, Penneçot GF, Bensahel H: Closed flexible intramedullary nailing of the femoral shaft fractures in children. J Pediatr Orthop B. 1997, 6:198-202. 10.1097/01202412-199707000-00008

9. Andreacchio A, Alberghina F, Marengo L, Canavese F: Pediatric tibia and femur fractures in patients weighing more than $50 \mathrm{~kg}(110 \mathrm{lb})$ : mini-review on current treatment options and outcome. Musculoskelet Surg. 2019, 103:23-30. 10.1007/s12306-018-0570-8

10. Chapman T, Sholukha V, Semal P, Louryan S, Rooze M, Van Sint Jan S: Femoral curvature variability in modern humans using three-dimensional quadric surface fitting. Surg Radiol Anat. 2015, 37:1169-77. 10.1007/s00276-015-1495-7 


\section{Cureus}

11. Egol KA, Chang EY, Cvitkovic J, Kummer FJ, Koval KJ: Mismatch of current intramedullary nails with the anterior bow of the femur. J Orthop Trauma. 2004, 18:410-5. 10.1097/00005131-200408000-00003

12. Greville NR, Ivins JC: Fractures of the femur in children; an analysis of their effect on the subsequent length of both bones of the lower limb. Am J Surg. 1957, 93:376-84. 10.1016/0002-9610(57)90826-7

13. Reynolds DA: Growth changes in fractured long-bones: a study of 126 children . J Bone Joint Surg Br. 1981, 63:83-8. https://doi.org/10.1302/0301-620X.63B1.7204480

14. Clement DA, Colton CL: Overgrowth of the femur after fracture in childhood. An increased effect in boys . J Bone Joint Surg Br. 1986, 68:534-6. 10.1302/0301-620X.68B4.3733825

15. Narayanan UG, Hyman JE, Wainwright AM, Rang M, Alman BA: Complications of elastic stable intramedullary nail fixation of pediatric femoral fractures, and how to avoid them. J Pediatr Orthop. 2004, 24:363-9. 10.1097/00004694-200407000-00004

16. Sink EL, Gralla J, Repine M: Complications of pediatric femur fractures treated with titanium elastic nails: a comparison of fracture types. J Pediatr Orthop. 2005, 25:577-80. 10.1097/01.bpo.0000164872.44195.4f

17. Parsch KD: Modern trends in internal fixation of femoral shaft fractures in children. A critical review . J Pediatr Orthop B. 1997, 6:117-25. 10.1097/01202412-199704000-00007

18. Joeris A, Bansi G, Knorr P, Lieber J, Schalamon P, Slongo T: ESIN in femur fractures. Eur J Trauma Emerg Surg. 2005, 31:24-32. 10.1007/s00068-005-1075-3

19. Marengo L, Nasto LA, Michelis MB, Boero S: Elastic stable intramedullary nailing (ESIN) in paediatric femur and tibia shaft fractures: comparison between titanium and stainless steel nails. Injury. 2018, 49:S8-S11. 10.1016/j.injury.2018.09.049

20. Gulati DS, Kumar A: Limb length discrepancy following conservative management of femoral shaft fractures in children, clinical review and recommendations. J Evid Based Med Healthc. 2018, 5:1747-1750. 10.18410/jebmh/2018/366 